\title{
Rendimiento académico: una mirada desde la procrastinación y la motivación intrínseca*
}

\author{
Academic achievement: a view from procrastination \\ and intrinsic motivation
}

Desempenho acadêmico: uma visão de procrastinação e motivação intrínseca

\author{
Claudia Patricia Navarro Roldan** \\ Recibido 09. 03. 2016 • Arbitrado 26. 03. 2016 • Aprobado 24.04. 2016
}

\section{Resumen}

Introducción: La literatura no explica contundentemente el cambio en la varianza del desempeño académico y su impacto en los niveles de deserción. La pregunta de investigación fue ¿Cuáles son los predictores del rendimiento académico en los estudiantes universitarios? Objetivo: Explicar la varianza del rendimiento académico desde factores individuales: procrastinación académico, motivación intrínseca (morosidad, temor al fracaso y factores de carrera) y variables de control (genero, trabajar y semestre). Metodología: Se usó un diseño exploratorio de corte transversal. Se seleccionaron intencionalmente 129 estudiantes de psicología de la UPTC, quienes diligenciaron la Escala de Procras-

Los resultados de este artículo hacen parte de proyecto de prácticas residenciales del Programa de Psicología de la Universidad Pedagógica y Tecnológica de Colombia (UPTC) liderados por el Comité de Desarrollo Académico de la UPTC y el grupo de investigación Desarrollo Humano, Cognición y Educación en su línea investigativa Desarrollo y Cognición. Se reconoce y agradece el trabajo de Jennifer Stephanie Amador Salinas en el proceso de recolección de datos.

** Doctora en Psicología de la Universidad de los Andes, Magister en Psicología de la Universidad del Valle y Psicóloga de la Universidad del Norte. Actualmente, profesora de planta en la Universidad Pedagógica y Tecnológica de Colombia (Tunja, Colombia) en el área psicología educativa, líder del grupo de investigación Desarrollo Humano, Cognición y Educación, y Coordinadora del Comité Desarrollo Académico de la Escuela de Psicología de la UPTC, claudia.navarro@uptc.edu.co 
tinación General de Tuckman y la Escala de Motivación Intrínseca. Resultados: El mejor predictor del Rendimiento Académico fueron los Factores de Carrera, seguido del Semestre y la Morosidad. Conclusiones: Se sugirió revaluar la participación que la educación superior cumple en la decisión vocacional y profesional de los estudiantes, así como generar programas que incrementen sus niveles de autorregulación.

Palabras clave: procrastinación académica, motivación intrínseca, rendimiento académico, psicología, deserción.

\section{Abstract}

Introduction: The literature does not conclusively explain the change in the variance of academic achievement and their impact on levels of desertion. The research question was what are the predictors of academic achievement by college students? Goal: To explain the variance of academic achievement from individual factors: academic procrastination, intrinsic motivation (delinquency, fear of failure and career factors), and control variables (gender, work, and semester). Methodology: This exploratory study used a cross-sectional design. Intentionally, we selected 129 psychology students form UPTC, who fill out General Procrastination Scale Tuckman and Intrinsic Motivation Scale. Results: The best predictor of academic achievement was a Factors Carrera, followed by Semester and Delinquency. Conclusions: We suggested revalue the participation that higher education plays in vocational and professional choice of students and create programs to increase their levels of self-regulation.

Keywords: Academic procrastination, intrinsic motivation, academic achievement, psychology, desertion.

\section{Resumo}

Introdução: A literatura não explica com claridade a mudança na variância do desempenho acadêmico e seu impacto nos niveis de de- 
serção. A pergunta da pesquisa foi ¿Quais são os preditores do desempenho acadêmico nos estudantes universitários? Objetivo: Explicar a variância do desempenho acadêmico desde fatores individuais: procrastinação acadêmica, motivação intrínseca (morosidade, temor ao fracasso e fatores de carreira universitária) e variáveis de controle (gênero, trabalhar e período acadêmico). Metodologia: Foi usado um desenho exploratório de cunho transversal. Foram selecionados 129 estudantes de psicologia da UPTC de forma intencional. Estes estudantes preencheram a Escala de procrastinação Geral de Tuckman e a Escala de Motivação Intrínseca. Resultados: O melhor preditor do desempenho acadêmico foram os fatores de carrera, em seguida do período acadêmico e a morosidade. Conclusão: Sugiri-se re-avaliar a participação da educação superior na decisão vocacional e profesional dos estudantes e também como gerar programas que incremente o nivel de autorregulação dos estudantes.

Palavras-chave: procrastinação acadêmica, motivação intrínseca, desempenho acadêmico, psicologia, deserção.

\section{Introducción}

El rendimiento académico mide el logro que un estudiante obtiene frente a las demandas de los ambientes de aprendizaje en los cuales participa. En este sentido, obtener altas o bajas calificaciones en las asignaturas cursadas es un indicativo de la calidad no solo de la experiencia de aprendizaje del estudiante, sino también de la institución educativa que diseña los ambientes de aprendizaje. En consecuencia, en aras de la calidad educativa, las instituciones de educación superior premian a los estudiantes con altos rendimientos académicos a través de becas, pasantías u otros estímulos y sancionan a los estudiantes con bajo rendimiento con la pérdida definitiva de su cupo universitario. Por ejemplo, en la Universidad Pedagógica y Tecnológica de Colombia [UPTC], de donde procede la muestra de este estudio, un estudiante es desvinculado del sistema universitario cuando 
pierde por tercera vez la misma asignatura, cuando pierde dos veces las mismas dos asignaturas, cuando obtiene un promedio académico por debajo de dos (2 sobre 5), entre otros causales relacionadas con el rendimiento académico (UPTC, 1998, art. 80). Similarmente, todas las instituciones de educación superior reglamentan diferentes requerimientos mínimos de rendimiento académico, medido en calificaciones numéricas, que aseguran o no la permanencia de un estudiante dentro del sistema universitario.

La permanencia exitosa de los estudiantes en las instituciones de educación superior colombianas se ha convertido en un factor tan importante que, en la actualidad, prevenir la deserción de los estudiantes del sistema universitario hace parte de los indicadores de calidad con los cuales son medidos los programas y las instituciones (MEN, 2010, art. 6,5; CNA, 2013, caract. 32). En consecuencia, cobra relevancia estudiar los determinantes académicos de la deserción universitaria y los elementos claves para generar estrategias que prevengan este tipo de deserción.

La deserción universitaria, vista como el abandono de la institución educativa durante dos periodos o semestres consecutivos (Sánchez, Quirós, Reverón y Rodríguez, 2002) debería generar alarma a nivel político y educativo en nuestra sociedad. El Ministerio de Educación Colombiana reportó una tasa de deserción acumulada del sistema universitario del 50\% de la población nacional para el 2014. Este estudio incluyó la información del Sistema para la Prevención de la Deserción de la Educación Superior (1998-01 hasta 2013-02), los resultados de las pruebas de conocimiento estatales para estudiantes de último año de secundaria Icfes Saber 11, entre otros, que alcanzan un aproximado de 4.018.254 registros (MEN, 2014).

En general, lo que muestra este estudio es que de cada dos estudiantes que ingresan al sistema universitario, tan solo uno de ellos logra graduarse. Adicionalmente, la caracterización reveló que el estudiante posee mayor probabilidad de desertar del sistema universitario cuando 
es hombre, trabaja, se encuentra entre los 21 y 25 años, pertenece a familias con muy bajos (o- 1 salario mínimo) o muy altos (5-7salarios mínimos o más) ingresos económicos, bajos niveles de educación de la madre, bajas puntuaciones en la prueba de estado Icfes Saber11, y más semestres cursados (MEN, 2014). Las razones atribuibles a estos altos niveles de deserción se derivan de la conjunción de causales económicas (e.g., no tener beca), sociales (e.g., familia desempleada, padres con bajos niveles educativos), individuales (e.g., porcentaje de repitencia de asignaturas) e institucionales (e.g., programas de apoyo al estudiante). El informe del MEN (2014) también reveló que a nivel nacional las tasas de deserción más altas superan el $45 \%$, y se encuentran en carreras tales como agronomía, veterinaria y afines, seguidos de ingeniería, arquitectura, urbanismo y afines. Por su parte, a nivel nacional, las carreas relacionadas con las ciencias de la salud (e.g., medicina, enfermería, odontología, psicología, entre otras) también presentan altos niveles de deserción, ya que alcanzan una tasa del $40 \%$.

En el departamento de Boyacá (donde se ubica la UPTC), las tasas de deserción aumentan a medida que el estudiante cursa más semestres. Por ejemplo, para cinco semestres cursados la tasa de deserción acumulada alcanza el 38,78\%, para diez semestres alcanza el 45,22\% y para 15 semestres cursados alcanza el 48,99\%. Específicamente, en la UPTC, entre 1998 y el 2010, se encontró que entre el primer y tercer semestre académico la tasa de deserción asciende al 32\%, y hasta quinto semestre se estabilizó en el $37 \%$. Al cabo de diez semestres académicos la tasa deserción en promedio llegó al 41\% (UPTC, 2011). Este estudio reveló que tienen mayor probabilidad de desertar de la universidad los estudiantes hombres, mayores de 24 años, con ingresos económicos altos, bajos resultados en las pruebas estatales Icfes Saber 11, y aquellos que no reciben apoyos económicos (e.g., becas). Adicionalmente, se reveló que tienen más riesgo de desertar los estudiantes de carreras presenciales tales como artes, agronomía, matemáticas, ciencias naturales y economía, y quienes cursan carreras tecnológicas en modalidad a Distancia. 
Específicamente, la carrera de psicología de la UPTC (muestra de este estudio) agrupa, en promedio, 350 estudiantes semestralmente. Los reportes institucionales revelan que, entre el 2009 y el 2014, las tasas de deserción semestral se ubicaron entre puntuaciones mínimas de 1,1\% y máximas del 4,5\%. Estos "bajos" porcentajes implican una voz de alarma, ya que en un período de cinco años, aproximadamente, 120 estudiantes han desertado de la carrera, y el 50\% de ellos han perdido el cupo universitario por causales relacionadas con el bajo rendimiento académico. Esto ocurre a pesar de que el sistema de selección de estos estudiantes se concentra en la obtención de niveles superiores de rendimiento en las pruebas de conocimiento estatales (i.e. Icfes Saber 11), las cuales, aparentemente, revelarían competencias cognitivas adecuadas para el ingreso a la universidad y un menor nivel de riesgo de deserción. Incluso, a pesar de que institucionalmente existen políticas de apoyo por incapacidad económica a través de becas, acompañamiento psicológico y educativo a los estudiantes con bajo rendimiento, y un programa de "padrinos" que involucra a estudiantes con altos rendimientos que apoyan el proceso académico de los estudiantes con bajo rendimiento.

En esta línea, en donde el bajo rendimiento académico es la causa que explica el 50\% de los casos de la deserción de los estudiantes de psicología de la UPTC, la pregunta que orientó este estudio fue ċcuáles son los predictores del rendimiento académico de los estudiantes universitarios? La evidencia empírica revela que no solo factores cognitivos explican el bajo rendimiento (Pérez y Cabanach, 1998; Pérez, 2007; Pérez, Ramón ySánchez, 2000); por el contrario, el bajo rendimiento puede ser explicado desde los factores motivacionales (Cabanach, Pérez, Martínez y Aguín, 1999; Núñez, Cabanach, Rodríguez, González y Rosário, 2009; Pérez, Ramón y Sánchez, 2000;) y comportamentales de los estudiantes (Digdon \& Howell, 2008; Dietz, Hofer \& Fries, 2007; Steel, 2007; Steel $\&$ Wambach, 2001), ya que estos se encuentran asociadas a problemas como el fracaso escolar y la deserción del sistema educativo.

A pesar de que existe evidencia empírica que revela múltiples causales del bajo rendimiento, los modelos existentes no ofrecen aún explicaciones contundentes sobre los cambios en la varianza del desempe- 
ño académico de los estudiantes universitarios. Este estudio pretende aportar evidencia en esta dirección. En consecuencia, se analizó un modelo de regresión que pretende predecir los cambios en el rendimiento académico de los estudiantes de psicología activos durante el segundo semestre del 2014 en la UPTC. Para ello, se evaluaron predictores individuales tales como los patrones de comportamiento procrastinador (académico) y la motivación intrínseca (desde la morosidad, temor al fracaso y factores de carrera). El modelo incluyó otras variables de control tales como el género, trabajar para sostenerse económicamente y el número de semestres cursados. Esta información resultó valiosa en la medida en que aportó insumos para generar futuras investigaciones e intervenciones que promuevan el éxito académico de estudiantes universitarios de psicología y, por tanto, disminuyan la deserción universitaria derivada del bajo rendimiento académico.

\section{Fundamentación teórica}

A continuación se describen los factores individuales analizados en este estudio. En primer lugar, la procrastinación general es un patrón comportamental generalizado caracterizado por el actuar (voluntario o involuntario) que demora y/o posterga el curso de las acciones programadas (Ariely \& Wertenbroch, 2002; Stell, 2007; Steel \& Wambach, 2001). Esto es, comportamientos que llevan al sujeto a demorar y aplazar las tareas cotidianas que deberían ser completadas (e.g., lavar los platos, solicitar una cita médica, depositar los ahorros en el banco), y al hacerlo, él pierde la motivación para realizarlas (Jackson, Weiss, Lundquist \& Hooper, 2003). La evidencia empírica revela que la adolescencia es el período del ciclo vital de mayor sensibilidad a comportarse de forma procrastinadora. En particular, algunos estudios indican que el 95\% de los adolescentes (aproximadamente) reportan comportamientos procrastinadores y un deseo de reducirlos. Sin embargo, al no lograrlo, ellos desarrollan un estado de angustia o auto-culpa que, a su vez, se traduce en malos indicadores de salud mental y bajos rendimientos académicos y laborales (Ariely \& Wertenbroch, 2002; Stell, 2007, Steel \& Wambach, 2001). 
Dentro de los contextos educativos, Ferrari (2001) argumenta que la procrastinación académica es el comportamiento evitativo de las responsabilidades y actividades académicas debido, tal vez, a que estas son percibidas como aversivas. Por ejemplo, prometer hacer las tareas más tarde, excusar o justificar los retrasos en la realización de las tareas, y evitar la culpa frente a la no realización de una tarea académica (Domínguez, Villegas y Centeno, 2014; Glick, Millstein \& Orsillo, 2014; Glick \& Orsillo, 2015; Onwuegbuzie, 2004; Quant y Sánchez, 2012). O’Brien (2002) encontró que entre el 80\% y el 95\% de los estudiantes universitarios reportaron comportamientos procrastinadores. Estos comportamientos también pueden estar relacionados con postergar las actividades académicas a través de la realización de otras actividades tales como ver televisión, dormir o jugar (Chun Chu \& Choi, 2005; Pychyl, Lee, Thibodeau \& Blunt, 2000). Incluso, procrastinar puede estar asociado con los pensamientos de ineficacia del estudiante, ya que estos impactan negativamente la consecución y el desarrollo de la actividad académica. Algunos de los factores determinantes del comportamiento procrastinador son el sentimiento de baja responsabilidad o poca concentración, los pensamientos de desamparo, la ansiedad y factores de la personalidad que influyen en la dilación académica (Balkıs \& Duru, 2009).

Consistentemente, diferentes estudios en contextos universitarios muestran la prevalencia de patrones de comportamiento procrastinador. Por ejemplo, investigaciones en estudiantes norteamericanos muestran que de 104 estudiantes, el 20\% de ellos reportan procrastinación académica crónica, y el 50\% se auto percibe como procrastinador. Los estudiantes reportan conductas asociadas a la procrastinación en más del 80\% de los casos (e.g., no cumplir el plan previsto, dejar para último momento la realización de una tarea planeada con anticipación); y en el 70\% de los casos, los estudiantes reportaron deseo de cambio de estas conductas como una forma de obtener éxito académico (Tice \& Baumeister, 1997). Resultados similares se encontraron en 580 futuros docentes de la Universidad de Pamukkale de Turquía, entre los 18 y 28 años, quienes en el 23\% de los casos presentaron altos niveles 
de procrastinación, y en el 27\% de los casos presentaron procrastinación moderada (Balkis \& Duru, 2009). En este estudio, se encontró que los hombres procrastinan con mayor frecuencia $(\mathrm{p}<\mathrm{0}, 01), \mathrm{y}$ los estudiantes entre los 13 y 18 años procrastinan más, en comparación con aquellos ubicados entre los 19 y 28 años. Otros estudios muestran que la procrastinación académica en la educación superior se relaciona con el rendimiento académico. Chan (2011) analizó 200 estudiantes de la Universidad de Lima, entre los 19 y 23 años. Los hallazgos revelaron que los estudiantes de alto y bajo rendimiento académico reportaron conductas procrastinadoras, pero aquellos que obtienen puntuaciones promedio más altas en procrastinación (i.e. 61,5\%) reportaron haber reprobado entre uno y seis cursos o materias durante el proceso de formación académica.

En segundo lugar, la motivación vista como el conjunto de procesos implicados en la activación, dirección y persistencia de la conducta (Beltrán, 1993; Good \& Brophy, 1983) se convierte en un factor clave para explicar el éxito o el fracaso académico. Diversos estudios muestran la asociación entre los procesos de motivación y los resultados de los procesos de aprendizaje, de forma tal que valoraciones positivas frente a las tareas académicas podrían conducir al estudiante a involucrarse más en el propio aprendizaje y a utilizar estrategias cognitivas de forma más frecuente (Pintrich, 2004; Pintrich \& De Groot, 1990; Schiefele, 1991; Wolters \& Pintrich, 1998). En este sentido, el interés o la apatía que el estudiante percibe ante las tareas académicas posibilita que el estudiante realice más o menos esfuerzo (respectivamente) en la realización de sus tareas, lo cual afecta a su vez el rendimiento académico obtenido.

En particular, la motivación intrínseca es la tendencia inherente del ser humano a realizar actividades por el placer de hacerlas, sin aparentes razones externas al individuo. Algunos elementos asociadas a la motivación intrínseca son la curiosidad, la competencia, el reconocimiento, la aceptación, la autonomía y los intereses (Cirino, 2003). 
Aguilar, Valencia, Martínez, Romero y Lemus (2004) argumentan que, en contextos académicos universitarios, la motivación intrínseca es el disfrute y gusto por las actividades escolares, el interés y el deseo de aprender y adquirir nuevos conocimientos. En esta línea, bajos niveles de motivación intrínseca en los estudiantes universitarios han sido asociados con altos niveles de ausentismo académico, bajas calificaciones, incremento en el número de materias repetidas y al abandono de los estudios universitarios (Pérez, Ramón y Sánchez, 2000). En este marco, la motivación intrínseca en los estudiantes universitarios es un constructo fundamental para reconocer el interés o la apatía por hacer una actividad académica desafiante, la cual es considerada un fin sí misma y no un medio para alcanzar otras metas (Lepper, 1988).

En síntesis, el rendimiento académico se ve favorecido por factores relacionados con el comportamiento y la motivación. No obstante, la evidencia existente aun no es concluyente con respecto a por qué altos porcentaje de estudiantes que ingresan al sistema universitario obtienen bajos rendimientos académicos. En este estudio se pretende aportar evidencia que analiza de forma articulada elementos derivados del comportamiento y la motivación de los estudiantes, y cómo estos logran o no explicar el rendimiento académico.

\section{Método \\ Participantes}

Participaron 129 estudiantes de la carrera de psicología de la UPTC, 104 fueron mujeres y 25 hombres, con una edad promedio de 20,27 años (D.E=2,91). El criterio de inclusión en la muestra fue estar matriculado como estudiante de psicología en la UPTC, que es un programa diurno y presencial. Adicionalmente, estar cursando primer, segundo y tercer semestre porque, históricamente, estos cursos revelan el mayor índice de deserción y repitencia de asignaturas, y pertenecer a octavo, noveno y décimo semestre porque estos cursos revelan el menor nivel de deserción y menor nivel de repitencia de asignaturas. En este sentido, intencionalmente se realizó una invitación abierta a los estudiantes 
en sus salones de clases, y voluntariamente los estudiantes participaron en el estudio.

\section{Instrumentos}

\section{Escala de procrastinación general de Tuckman (ATPS)}

Se usó la Escala de Procrastinación General de Tuckman (Tuckman, 1990) en la versión validada con estudiantes universitarios argentinos (Furlan, Heredia, Piemontesi y Tuckman, 2012). La escala quedó conformada por 15 reactivos que reportaron ser altamente constantes $(\alpha=0,87)$, los cuales evalúan la tendencia general a perder el tiempo y postergar la realización de actividades (e.g., "cuando tengo un plazo, espero hasta el último momento para realizarlo; derrocho mucho tiempo y me parece que no puedo hacer nada al respecto"). Cada reactivo cuenta con cinco opciones de respuesta de frecuencia que van desde nunca hasta siempre. Los autores reportan estudios complementarios de validación que ofrecieron evidencias de test criterio, con correlaciones negativas (- .217, p<.001) con el rendimiento académico. Adicionalmente, las correlaciones parciales con las dimensiones de la ansiedad frente a los exámenes demuestran un patrón diferencial para cada una de ellas.

\section{Escala de Motivación Intrínseca}

Esta escala mexicana Aguilar, Valencia, Martínez, Romero y Lemus, 2004; Aguilar, Martínez, Valencia, Romero y Vargas, 2001) validada en estudiantes de la Universidad Pedagógica y Tecnológica de Colombia (Ardila, Sanabria y Cárdenas, 2010). El análisis factorial rotado ( $\mathrm{KMO}=0,901$; Barllet $\left(X_{(820)}=11602,202, \mathrm{p}<0,01\right)$ permitió constituir un cuestionario con 38 reactivos, los cuales reportaron ser altamente constante $(\alpha=0,875)$. La escala fue conformada por tres escalas psicométricas, todas con reactivos de cuatro opciones de respuesta que van desde completamente en desacuerdo hasta completamente de acuerdo.

Las escalas son: a) Morosidad con 9 reactivos que valoran el sentimiento de desagrado o disgusto en la realización y entrega de las tareas 
académicas (e.g., "cuando tengo que hacer algo importante pero que no me gusta, me toma mucho tiempo empezar hacerlo; soy un poco indiferente para realizar mis deberes y las cosas que me interesan”); b) Temor al fracaso con 11 reactivos que evalúan la insatisfacción con el propio desempeño y la reacción excesiva a exagerar los propios errores (e.g., "cuando encuentro errores en mis tareas reacciono como si todo hubiese quedado mal; siento como un fracaso cuando las tareas no quedan como yo me lo había propuesto"); y c) Factores de carrera con 18 reactivos que evalúan la satisfacción percibida por parte del estudiante frente a la certeza de la decisión vocacional y las competencias percibidas para realizar las actividades académicas de la carrera seleccionada. En esta escala se agruparon factores relacionados con la autoeficacia, el autoconocimiento, la información sobre la carrera y la ansiedad ante la elección de la carrera (e.g., "para que pueda decidir si continúo o no estudiando la carrera que actualmente estudio, todavía necesito recibir orientación sobre qué carrera me interesaría y en cual podría desempañarme bien; me parece que mis intereses no son lo suficientemente fuertes para seguir estudiando mi carrera profesional").

\section{Diseño y procedimiento}

En este estudio exploratorio, de corte transversal, se aplicó de forma grupal la Escala de Motivación Intrinseca y la Escala de Procrastinación General de Tuckman a los 129 estudiantes de psicología que consintieron voluntariamente su participación. El promedio de calificación semestral de los estudiantes se obtuvo del sistema información y registro académico de la UPTC. Los datos fueron analizados usando el SPSS 21, el cual permitió realizar un modelo de regresión lineal múltiple, con un nivel de significancia de 0,05, para establecer sí el rendimiento académico de los estudiantes de psicología se predice desde el modelo diseñado en este estudio. Adicionalmente, se realizaron comparaciones de medias y de puntuaciones promedio para revelar el comportamiento de los predictores incluidos en el modelo (i.e., rendimiento académico, procrastinación académica y motivación intrínseca) a partir de las variables de control (i.e., semestre académico, género y trabajar). 


\section{Consideraciones éticas}

En este estudio considerado de bajo riesgo, los estudiantes participaron voluntariamente y diligenciaron el consentimiento informado. Adicionalmente, para salvaguardar el anonimato y la confidencialidad de los datos de los participantes sus nombres fueron remplazados por un código en la base de datos. Esto es, toda la información suministrada, facilitada, colectada, conservada y procesada, durante la ejecución de este estudio, fue utilizada por las partes con estricto cumplimiento de lo preceptuado en las normas constitucionales, legales y desarrollos en materia de protección de 'Datos personales - Data protections - Habeas data' (Minsalud, 1993, Res. 8430; Ley 1581 de 2012; Mincomercio, 2014, Dec. 1317; COLPSIC, 2012).

\section{Resultados}

En primer lugar, se analizó el comportamiento de las variables objeto de estudio (i.e., rendimiento académico, procrastinación académica y motivación intrínseca) con respecto a las variables de control (i.e., semestre académico, género y trabajar). Posteriormente, se presenta el modelo de regresión que logró predecir el 35\% de la varianza del Rendimiento Académico de los estudiantes de psicología analizados.

\section{Comportamiento de las variables objeto de estudio con respecto a las variables de control \\ Variaciones de acuerdo al semestre académico}

En primer lugar, se analizó el rendimiento académico de los estudiantes. El análisis de varianza arrojó que existen diferencias estadísticamente significativas entre las puntuaciones promedio del rendimiento académico y el semestre cursado $\left(F_{(5,123)}=12,039, \mathrm{p}=0,00\right)$. La prueba pos hoc (Prueba Bonferroni<0,05) indicaron diferencias significativas entre las calificaciones semestrales promedio de primer semestre y los semestres restantes. Específicamente, en primer semestre los estudiantes obtuvieron puntuaciones promedio menores, y fueron los estudiantes de décimo semestre quienes obtuvieron rendimientos académicos más altos (ver Tabla 1). 


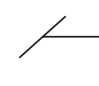

En segundo lugar, el análisis mostró que las puntuaciones promedio de procrastinación académica difieren entre los diferentes semestre ( $\mathrm{F}$ $(5,124)=2,784, \mathrm{p}=0,02)$. La prueba pos hoc (Prueba Bonferreni<0,05) indicó que las puntuaciones promedio difieren estadísticamente entre primer y décimo semestre (ver Tabla 2), ya que los estudiantes de décimo presentaron puntuaciones promedio mayores $(\mathrm{M}=0,61 ; \mathrm{DE}=1,53)$, en comparación con las puntuaciones de los estudiantes de primer semestre $(M=-0,23 ; D E=0,54)$. Adicionalmente, la procrastinación académica difiere estadísticamente $(\mathrm{p}<0,05)$ entre octavo y décimo semestre, ya que en décimo se obtuvieron puntuaciones promedio mayores que en octavo $(\mathrm{M}=-0,33 ; \mathrm{DE}=0,01)$.

Tabla 1: Distribuciones de predictores del rendimiento académico de acuerdo al semestre cursado.

\begin{tabular}{|lccccc|}
\hline Semestre cursado & N & \multicolumn{2}{c}{$\begin{array}{c}\text { Rendimiento } \\
\text { académico }\end{array}$} & \multicolumn{2}{c|}{$\begin{array}{c}\text { Procrastinación } \\
\text { Académica }\end{array}$} \\
\hline & & Media & DE & Media & DE \\
\hline Primero & 43 & 3,65 & 0,34 & $-0,23$ & 0,54 \\
\hline Segundo & 2 & 3,85 & 0,07 & $-0,32$ & 0,008 \\
\hline Tercero & 29 & 4,02 & 0,23 & 0,02 & 1,07 \\
\hline Octavo & 24 & 3,97 & 0,27 & $-0,33$ & 0,019 \\
\hline Noveno & 17 & 4,26 & 0,50 & 0,28 & 1,32 \\
\hline Décimo & 14 & 4,32 & 0,47 & 0,61 & 1,53 \\
\hline Total & 129 & 3,95 & 0,42 & $-0,02$ & 0,96 \\
\hline
\end{tabular}

Fuente: propia. Las calificaciones posibles se encuentran entre 0.0 hasta 5.0, siendo esta última el más alto rendimiento académico posible.

Para finalizar, el análisis de varianza para la variable de motivación (i.e., morosidad, temor al fracaso y factores de la carrera) no reveló diferencias significativas entre los semestres ( $p>0,05)$. No obstante, el análisis descriptivo de las puntuaciones promedio de los estudiantes de octavo grado mostró comportamientos diferentes a los semestres restantes (ver Tabla 2). Ellos obtuvieron puntuaciones promedio mayores en morosidad, temor al fracaso y factores de carrera. Esto podría 
estar relacionado con que octavo (en la muestra objeto de estudio) es el último semestre teórico antes de ingresar a las prácticas residenciales del último año de carrera, lo cual podría impactar las expectativas favorables de los estudiantes ante la culminación de la carrera y el ingreso a las prácticas profesionales lo que, a su vez, favorecería el incremento en el nivel de satisfacción percibida con la carrera de psicología. Sin embargo, por otro lado, la alta demanda teórica que posee octavo semestre (en donde cursan psicología clínica, educativa, organizacional y comunitaria, cada una con intensidad de 9 horas semanales) podría estar relacionada con que los estudiantes no terminen los trabajos y tareas exigidas y, por tanto, sientan temor ante el fracaso académico. Resultados similares se observan en Aguilar, Martínez, Valencia, Conroy \& Girardo (1997) y Eliot \& Church (1997) quienes argumentan la asociación positiva entre la morosidad y ansiedad evaluativa, la evitación en la presentación de trabajos dentro del tiempo institucionalmente establecidos. En suma, el conjunto de factores personales y contextuales podrían estar explicando los cambios en los niveles generales de motivación en los estudiantes de octavo semestre, en comparación con las puntuaciones promedio obtenidas por los estudiantes de los semestres restantes.

Tabla 2: Distribuciones promedio de factores motivaciones de acuerdo al semestre cursado.

\begin{tabular}{|c|c|c|c|c|c|c|c|}
\hline $\begin{array}{c}\text { Semestre } \\
\text { cursado }\end{array}$ & N & \multicolumn{2}{|c|}{$\begin{array}{c}\text { Morosidad } \\
\end{array}$} & & \multicolumn{2}{c|}{$\begin{array}{c}\text { Temor al } \\
\text { fracaso }\end{array}$} & \multicolumn{2}{c|}{$\begin{array}{c}\text { Factores de } \\
\text { carrera }\end{array}$} \\
\hline & 43 & $-0,10$ & 0,05 & $-0,08$ & 0,05 & $-0,09$ & 0,04 \\
\hline Primero & 2 & $-0,08$ & 0,01 & $-0,10$ & 0,007 & $-0,12$ & 0,03 \\
\hline Segundo & 29 & $-0,06$ & 0,29 & $-0,05$ & 0,22 & $-0,04$ & 0,13 \\
\hline Tercero & 24 & 0,33 & 2,24 & 0,35 & 2,25 & 0,36 & 2,29 \\
\hline Octavo & 17 & $-0,02$ & 0,33 & $-0,09$ & 0,06 & $-0,11$ & 0,03 \\
\hline Noveno & 14 & $-0,10$ & 0,05 & $-0,12$ & 0,04 & $-0,07$ & 0,18 \\
\hline Décimo & 129 & 0,0008 & 1,00 & 0,001 & 1,00 & $-0,09$ & 0,04 \\
\hline Total & & & & & & & \\
\hline
\end{tabular}

Fuente: propia 


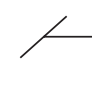

\section{Variaciones de acuerdo al género}

$\mathrm{El}$ análisis reportó diferencias estadísticamente significativas entre los hombres y las mujeres con respecto a la procrastinación académica $\left(t_{(104.56)}=3,668, \mathrm{p}=0,00\right)$, ya que las mujeres se reportaron como más procrastinadoras $(\mathrm{M}=0,68, \mathrm{DE}=1,08)$, en comparación con los hombres $(\mathrm{M}=-0,324, \mathrm{DE}=0,04)$. Por otra parte, frente a las variables motivacionales, se encontró que existen diferencias significativas en el temor al fracaso de los estudiantes $\left(t_{(121)}=-2,121, \mathrm{p}=0,036\right)$, ya que los hombres presentan mayores niveles en esta variable $(\mathrm{M}=-0,373, \mathrm{DE}=2,21)$ que las mujeres $(\mathrm{M}=-0,084, \mathrm{DE}=0,099)$. Para el resto de variables no se encontraron diferencias significativas de acuerdo al género $(\mathrm{p}>0,05)$.

Tabla 3: Distribución de puntuaciones promedio con respecto al género y trabajar.

\begin{tabular}{|c|c|c|c|c|c|c|c|c|c|}
\hline \multirow{2}{*}{\multicolumn{2}{|c|}{$\begin{array}{l}\text { Predictores } \\
\text { Media }\end{array}$}} & \multicolumn{2}{|c|}{$\begin{array}{l}\text { Hombres } \\
(\mathrm{n}=22)\end{array}$} & \multicolumn{2}{|c|}{$\begin{array}{l}\text { Mujeres } \\
(\mathrm{n}=105)\end{array}$} & \multicolumn{2}{|c|}{$\begin{array}{c}\text { Trabajan } \\
(\mathrm{n}=27)\end{array}$} & \multicolumn{2}{|c|}{$\begin{array}{c}\text { No trabajan } \\
(\mathrm{n}=103)\end{array}$} \\
\hline & & $\mathrm{DE}$ & Media & $\mathrm{DE}$ & Media & $\mathrm{DE}$ & Media & $\mathrm{DE}$ & \\
\hline $\begin{array}{l}\text { Procrastinació } \\
\text { Académica }\end{array}$ & & $-0,32$ & 0,04 & 0,06 & 1,08 & $-0,20$ & 0,64 & 0,05 & 1,07 \\
\hline \multirow{3}{*}{$\begin{array}{l}\text { Motivación } \\
\text { intrínseca }\end{array}$} & Morosidad & 0,32 & 2,20 & $-0,08$ & 0,20 & 0,35 & 2,12 & $-0,09$ & 0,15 \\
\hline & $\begin{array}{l}\text { Temor al } \\
\text { Fracaso }\end{array}$ & 0,37 & 2,21 & $-0,09$ & 0,05 & 0,31 & 2,12 & $-0,08$ & 0,11 \\
\hline & $\begin{array}{l}\text { Factores de } \\
\text { carrera }\end{array}$ & 0,32 & 2,20 & $-0,08$ & 0,09 & 0,34 & 2,15 & $-0,09$ & 0,07 \\
\hline $\begin{array}{l}\text { Rendimiento } \\
\text { semestral }\end{array}$ & académico & 3,83 & ,419 & 3,98 & 0,42 & 3,92 & 0,40 & 3,96 & 0,43 \\
\hline
\end{tabular}

Fuente: propia.

El análisis descriptivo de las puntuaciones promedio indicó que las mujeres presentan menos comportamientos procrastinadores académicos, menos morosidad y temor al fracaso y mayores promedios académicos, en comparación con los hombres. Sin embargo, ellas presentan menores niveles de factores de carrera, lo cual revela menor nivel de satisfacción percibida con la carrera seleccionada y menos nivel de capacidad percibida para cursarla, en comparación con los hombres. 


\section{Variaciones con respecto a la condición de trabaja mientras estudia}

Con respecto a la condición Trabaja/No trabaja para sostenerse económicamente, el análisis arrojó que existen diferencias estadísticamente significativas en la variable morosidad $\left(t_{(123)}=-2,119, \mathrm{p}=0,036\right)$; de forma tal que los estudiantes que trabajan muy a menudo no terminan sus actividades académicas $(\mathrm{M}=0,35, \mathrm{DE}=2,12)$, en comparación de aquellos que no trabajan ( $\mathrm{M}=-0,09 ; \mathrm{DE}=0,15)$; tal vez, porque cuentan con menos tiempo para cumplir con las demandas académicas de una carrera profesional de carácter diurna y presencial.

No obstante, en la variable factores de carrera $\left(t_{(120)}=-2,022, \mathrm{p}=0,04\right)$ quienes trabajan presentan mayor niveles de satisfacción y competencia percibida frente a la carrera que seleccionaron $(\mathrm{M}=0,34 ; \mathrm{DE}=2,15)$, en comparación de quienes no trabajan ( $\mathrm{M}=-0,09 ; \mathrm{DE}==, 07)$; estos hallazgos sugieren que los estudiantes que trabajan se informaron más sobre la carrera a estudiar y se perciben más capaces de asumir la demanda académica de la carrera. Para las variables restantes no se presentaron diferencias estadísticamente significativas ( $p>0,05)$.

\section{Modelo de regresión lineal múltiple del rendimiento académico}

En este estudio se realizó un análisis de regresión lineal múltiple, con un nivel de significancia del 0,05, para establecer sí el rendimiento académico de los estudiantes de psicología se explica desde la Procrastinación académica y la Motivación intrínseca (morosidad, temor al fracaso y factores de carrera). El modelo incluyó como variables de control la condición de trabajar (o no) mientras se estudia, género y semestre cursado. El análisis exploratorio preliminar indicó que las variables continuas del modelo se distribuyeron relativamente de forma normal, la prueba de Durbin y Watson (1.829) indicó que los residuos no están interconectados y, por tanto, se cumple el supuesto indicando homocesdasticidad. Por su parte, la prueba ómnibus muestra la utilidad del uso del modelo $\left(\mathrm{F}_{(7,123)}=9,620\right.$, $\mathrm{p}=0,00$ ). En la Tabla 4 se presentan los resultados del análisis, el cual revela que el modelo de regresión logró predecir el 35\% de la varianza del rendimiento académico de los estudiantes de psicología en la UPTC. 
Específicamente, el análisis del modelo de regresión encontró que los factores de carrera fueron el mejor predictor de la varianza del rendimiento académico $\left(\beta=1,549, \mathrm{t}=2,206, \mathrm{p}=0,03, \mathrm{r}^{2}=0,04\right)$; de forma tal que por cada unidad logarítmica en factores de carrera se logra predecir un incremento del rendimiento académico de 1,549 [IC 0,65; $1,22]$. Por otra parte, en el modelo también resultaron significativos otros predictores tales, por un lado, el semestre $(\beta=0,589, t=7,494$, $\left.\mathrm{p}=0,00, \mathrm{r}^{2}=0,34\right)$, es decir, por cada semestre académico cursado se logra predecir un incremento del rendimiento académico de 0,589 [IC $0,052 ; 0,89] ; y$, por otro, la morosidad $(\beta=-, 899, t=-2,195, p=0,03$, $\left.r^{2}=0,004\right)$, es decir, por cada unidad logarítmica en morosidad se logra predecir un decremento del rendimiento académico de 0,899 [IC -0,705;-0,036]. Los demás predictores del modelo resultaron no significativos (p>0,05). En suma, la ecuación que representa el modelo es:

Rendimiento Académico = Constante (3.666) + Factores de Carrera ${ }^{*} 1,549+$ Semestre cursado ${ }^{*} 0.589+$ Morosi$\mathrm{dad}^{*}-0.899$

Tabla 4: Análisis de regresión multivariada del rendimiento académico en estudiantes universitarios de psicología.

\begin{tabular}{|c|c|c|c|c|c|c|c|c|c|c|c|}
\hline 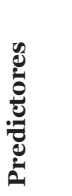 & 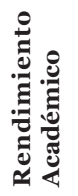 & 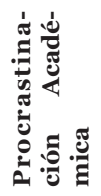 & 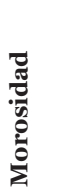 & 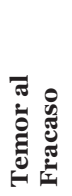 & 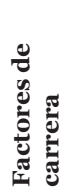 & b & DE & $\boldsymbol{\beta}$ & IC & & $\mathbf{S r}^{2}$ \\
\hline 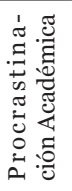 & 154 & & & & & ,002 & ,037 & ,005 &,- 072 & , 076 & 0,000 \\
\hline 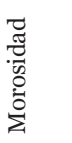 & ,011 &,- 041 & & & &,- 370 & 169, &,$- 899^{*}$ &,- 705 &,- 036 & 0,04 \\
\hline 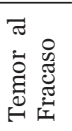 & , 026 &,- 037 & 979, & & &,- 262 & 276 &,- 630 &,- 810 & 286 & 0,008 \\
\hline
\end{tabular}




\begin{tabular}{|c|c|c|c|c|c|c|c|c|c|c|c|}
\hline 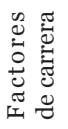 & ,039 & -,033 & ,981 & ,993 & & 647 & 293 & $1,549^{*}$ & ,065 & 1,22 & 0,04 \\
\hline 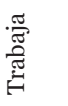 &,- 066 &,- 155 & ,197 & ,178 & , 180 &,- 116 & , o84 &,- 110 &,- 282 & ,051 & 0,017 \\
\hline 造 &,- 135 &,- 145 & 177 & ,201 & ,181 &,- 113 & , o87 &,- 104 &,- 286 & ,059 & 0,015 \\
\hline 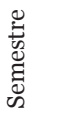 &, 568 & ,184 & ,082 & ,067 & ,064 & , 070 & ,009 &, $589^{* * *}$ & ,052 & , o89 & 0,34 \\
\hline 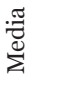 & 3,92 & $-0,05$ & 0,009 & 0,009 & 0,003 & & & & & & \\
\hline 됨 & 0,43 & 0,92 & 1,05 & 1,04 & 1,03 & $\begin{array}{l}\mathrm{R}{ }^{2} \\
=0,391^{* * *}\end{array}$ & \multicolumn{3}{|c|}{$\mathrm{R}^{2}$ Ajustado $=0,350^{* * *}$} & \multicolumn{2}{|c|}{$\begin{array}{l}\text { I n t e r c e p to } \\
=3,666^{* * *}\end{array}$} \\
\hline
\end{tabular}

Fuente: Propia. Variable Dependiente Rendimiento Académico Semestral. * $\mathrm{p}<0,05 ;{ }^{* *} \mathrm{p}<0,01 ;{ }^{* *} \mathrm{p}<0,001$.

\section{Conclusiones y Recomendaciones}

Los hallazgos del modelo de regresión revelaron que el incremento del rendimiento académico en los estudiantes de psicología, aquí analizados, se predice desde sentirse más satisfecho con la carrera seleccionada, haber cursado más semestres, y sentirse con agrado por la realización efectiva de trabajos académicos. En particular, los factores de carrera resultaron ser el mejor predictor del rendimiento académico, lo cual indica que cuando el estudiante tiene información sobre la carrera que estudia (en este caso sobre psicología), y se percibe más satisfecho y capaz de cursarla, entonces el estudiante logra obtener mejores resultados académicos. Por el contrario, si el estudiante se sintiera insatisfecho con la decisión vocacional, su rendimiento académico decrecería. Resultados similares revelan que los estudiantes insatisfechos con su decisión vocacional, generalmente, presentan falta de información sobre sí mismos (e.g., aptitudes, intereses) y sobre el ámbito laboral (e.g., 
salarios en el mercado laboral, opciones de trabajo) y poseen problemas en los procesos de solución de problemas (Gómez y Rivas, 1997; Maytorena, González y Velarde 2006).

En este sentido, que los Factores de carrera logren predecir el rendimiento académico involucra elementos que se derivan de la información que el estudiante tiene de la carrera seleccionada, del autoconocimiento que él tenga de sus propias habilidades para cursar la carrera y la correspondencia entre las características de la carrera seleccionada y sus habilidades (Cortina, 2003). Los factores de carrera también se encuentran relacionados con la compresión que el estudiante posee de elementos de la carrera tales como los planes de estudio, el tipo de materia que lo componen, la duración de la carrera, el modelo pedagógico privilegiado, la infraestructura disponible de laboratorios y salones, el reconocimiento de los lugares de práctica disponibles y de las perspectivas en el mundo laboral (i.e., profesiones, actividades vinculadas, posibilidades de trabajo, perspectivas económicas) (Cabello, 2001). Esto es, una decisión vocacional y profesional más informada permitiría que el estudiante obtenga mejores rendimientos académicos y, por tanto, un menor riesgo de desertar del sistema universitario.

En consecuencia, los sistemas de admisión a la universidad deberían, por un lado, generar diferentes experiencias que permiten al estudiante de último año de secundaria informarse ampliamente antes de tomar la decisión vocacional; y por otro, evaluar el impacto que este tipo de estrategias generan sobre el nivel de deserción universitaria. Generalmente, las instituciones de educación superior usan estrategias informativas a través de plegables, información en medios de comunicación, videos institucionales, entre otros, que informan sobre cuáles son las opciones de carrera, de qué se trata cada una de ellas, cuál es el perfil profesional y laboral que desarrollará, cuáles son las ocupaciones que podría desempeñar en el futuro, entre otros elementos (Robbins, Morrill \& Boggs, 1987). No obstante, este paquetes de medios de información deberían ir acompañados de otras estrategias tales como los procesos de orientación profesional y vocacional a los estudiantes de 
últimos años de secundaria; estos procesos de orientación podrían ser ofertados por los programas de psicología de las diferentes universidades del país como un programa de extensión social a la comunicad, o en su defecto, ser realizados por profesionales que los colegios contraten.

Adicionalmente a estas medidas, las instituciones de educación superior podrían implementar jornadas de "casa abierta" en donde se abran las puertas universitarias a los estudiantes de bachillerato para tomar cursos intersemestrales, conocer el funcionamiento pedagógico de la institución o, conocer más a fondo los programas que se ofrecen. Lo que se esperaría es que posteriormente se evalúe sí decisiones vocacionales más informadas se asociaron con altos niveles de satisfacción percibida con la carrera y, a su vez, con altos rendimientos académicos y menores niveles de deserción. Este tipo de resultados impactaría sobre la disminución de las pérdidas económicas y sociales generadas por la deserción de los estudiantes del sistema universitario.

En esta misma línea, sería importante implementar programas de psicoeducación dentro de los programas de psicología que orienten al estudiante activo sobre las posibles opciones laborales, y cómo estas se pueden relacionar o no con sus intereses vocacionales y laborales. Esto es, psicoeducar a los estudiantes con respecto a los perfiles profesionales tales como psicólogo jurídico, organizacional, educativo, publicitario, clínico infantil, familiar, entre otras múltiples opciones. Lo esperable sería que sí el estudiante se informará acerca de las ocupaciones y opciones laborales, entonces él podría orientar la selección de sus materias de profundización e incrementar su nivel de satisfacción percibida hacia la carrera; a su vez, esto podría favorecer el incremento de los niveles de rendimiento alcanzado por los estudiantes.

En suma, se sugiere que las políticas institucionales para disminuir la deserción universitaria, derivada de bajos rendimientos académicos, incremente el uso de programas que orienten a los estudiantes en la selección de la carrera que se estudiará, y luego otros programas que permitan orientar la selección de la línea de profundización profesional que ellos seguirán dentro de la carrera. Este tipo de políticas requiere 
procesos de intervención multidimensional que involucren favorecer los procesos de autoconocimiento de los estudiantes, mejorar los procesos de evaluación de alternativas y reflexión crítica en la toma de decisiones, evaluación de los programas información de las universidades, entre otros elementos.

Por otra parte, los hallazgos también revelaron que los estudiantes que presentaron menor nivel de morosidad, es decir que sienten gusto frente a la realización de sus tareas, obtuvieron más bajos rendimientos académicos. Por el contrario, quienes obtienen puntuaciones altas en morosidad sienten apatía frente a las tareas académicas y, en consecuencia, no sienten el impulso de realizarlas y/o entregarlas dentro de los tiempos estipulados. La literatura revela que altos niveles de morosidad ha sido asociada con altos niveles de ansiedad ante los exámenes y temor al fracaso, y bajos niveles de autorregulación y de autoeficacia (Aguilar y Valencia, 1994). No obstante, la morosidad también suele asociarse a variables contextuales que deberían ser tenidas en cuenta en futuras investigaciones, por ejemplo, ausencia de reglamentación educativa (e.g., no conocer los contenidos programáticos y cronograma de la asignatura) y altas demandas académicas (e.g., requerimientos para una tesis); incluso, puede ser generada por causas de fuerza mayor del estudiante (e.g., enfermedad, muerte de un familiar).

En esta línea argumentativa, se sugiere generar programas de intervención que vinculen la autorregulación como el elemento clave para articular los factores afectivos y motivacionales, con factores comportamentales y contextuales (Pintrich, 2004; Schwinger, Steinmayr \& Spinath, 2009). El trabajo sobre los procesos de autorregulación en los estudiantes universitarios posibilitaría generar motivos y afectos que disparen y direccionen comportamientos que incrementen el rendimiento académico. Por ejemplo, asistir a las clases que no le gustan, estudiar asignaturas que no le gustan con el mismo esfuerzo que aquellas que le gustan, entregar los trabajos dentro de los tiempos estipulados, controlar los niveles de ansiedad frente a los exámenes, no exagerar la valoración negativa de los errores cometidos, planear la realización de 
actividades para poder cumplir con las demandas académicas, entre otros elementos.

Siguiendo a Valle, González, Nuñez, Martínez y Pineñor (2008), la autorregulación emocional, cognitiva y comportamental es un proceso activo, en el cual el estudiante establece sus objetivos principales de aprendizaje y, a lo largo de este, trata de conocer, controlar y regular sus cogniciones, motivaciones y comportamientos de cara a alcanzar sus objetivos académicos. Lo esperable es que sí el estudiante aprende a regular el esfuerzo que implica las diferentes tareas académicas (i.e., es decir, la tendencia a mantener el foco y el esfuerzo hacia las metas, a pesar de las posibles distracciones), entonces él será capaz de lograr resultados satisfactorios en los plazos previstos (Chen, 2002; Corno, 1994; Kim \& Seo, 2013). Se sugiere realizar este tipo programas desde el primer semestre académico, ya que históricamente estos estudiantes se encuentran en un alto nivel de riesgo de deserción y, son ellos quienes presentan más bajos niveles de rendimiento académico.

Para finalizar, el modelo de regresión analizado reveló que entre más semestre el estudiante cursa se incrementa el rendimiento académico. Esto podría estar relacionado con que el estudiante, tal vez, logra ajustarse a la estructura del programa curricular cursado y se siente más satisfecho con su carrera. No obstante, otros elementos no considerados en este estudio podrían estar asociados a estos rendimientos académicos más altos. Por ejemplo, en los últimos semestres académicos de psicología, el currículo involucra más asignaturas de carácter teórico práctico o prácticas que evalúan el desempeño y competencias de los estudiantes de formas distintas a la evaluación en los semestres restantes. Por ejemplo, mientras que en las prácticas profesionales de ultimo año se evalúa la capacidad para solucionar problemas en las relaciones interpersonales, capacidad para formular un programa de intervención, desarrollo de habilidades terapéuticas, entre otros; en los años de carrera restantes las asignaturas evalúan a través de parciales memorísticos, solución de problemas hipotéticos, redacción de ensayos e informes de investigación, entro otros. En este sentido, las formas 
de evaluación y sus objetivos podrían tener un impacto en la obtención de bajos o altos rendimientos académicos en los estudiantes.

En síntesis, este estudio encontró que mejores rendimientos académicos se obtienen cuando el estudiante universitario de psicología se siente más satisfecho con la carrera seleccionada y se siente capaz de cursar la carrera, ha cursado más semestres y siente agrado por las realizaron y entrega efectiva de tareas académicas. En consecuencia, se sugirió revaluar la participación que las instituciones de educación superior cumplen en los procesos de orientación vocacional y profesional, así como generar programas que incrementen los niveles de autorregulación de los estudiantes universitarios.

\section{Referencias}

Aguilar, J., Valencia, A. (1994). Medición e interrelaciones entre temor al fracaso y morosidad. Revista de Psicología Social y Personalidad, 1o(2), 145-155.

Aguilar, J., Martínez, M., Valencia, A., Conroy, C., Girado, A. (1997). Metas de logro, competitividad y perfiles motivacionales entre estudiantes universitarios. Revista Latina del Pensamiento y Lenguaje, 5(1), 25-35.

Aguilar, J., Martínez, M., Valencia, A., Romero, P., Vargas, V. (2001). Interpretaciones de factores asociados a la motivación intrínseca. Revista Mexicana de Psicología, 18(2), 265-272.

Aguilar, J., Valencia, A., Martínez, M., Romero, P., Lemus, L. (2004). Estilos parentales y medidas de desarrollo psicosocial en estudiantes universitarios. Revista Latina de Pensamiento y Lenguaje, 12(1), 69-81.

Ardila, Y., Sanabria, L., Cárdenas, L. (2010). Estandarización de un modelo de motivación intrínseca con estudiantes de la Universidad Pedagógica y Tecnológica de Colombia. (Tesis de pregrado para optar por el título de psicólogo). Universidad Pedagógica y Tecnológica de Colombia, Tunja-Colombia. 
Ariely, D., Wertenbroch, K. (2002). Procrastination, deadlines, and performance: self-control by precommitment. Psychological Science, 13(3), 219-224. doi: 10.1111/1467-9280.00441.

Balk1s, M., Duru, E. (2009). Prevalence of academic procrastination behavior among preservice teachers, and its relationships with demographics and individual preferences. Journal of Theory and Practice in Education, 5(1), 18-32. Recuperado de http://eku.comu. edu.tr/index/5/1/mbalkis_eduru.pdf.

Beltrán, J. (1993). Procesos, estrategias y técnicas de Procesos, estrategias y técnicas de aprendizaje. Síntesis: Madrid.

Cabanach, R. G., Pérez, J. C. N., Martínez, S. R., Aguín, I. P. (1999). Un modelo causal sobre los determinantes cognitivo-motivacionales del rendimiento académico. Revista de psicología general y aplicada: Revista de la Federación Española de Asociaciones de Psicología, 52(4), 499-519. Recuperado de file://C:/ Users/CAROLINA.LATORRE/Downloads/Dialnet-UnModeloCausalSobreLosDeterminantesCognitivomotiva-2498648\%20(3).pdf.

Cabello, C. (2001). La cultura en la teoría de los recursos y capacidades: Una aproximación empírica. XI Congreso Nacional de ACEDE, Zaragoza.

Chan, L. (2011). Procrastinación académica como predictor en el rendimiento académico en jóvenes de educación superior. Temática Psicológica, 7(1), 53-62. Disponible en http://www.unife.edu.pe/publicaciones/revistas/revista_tematica_psicologia_2011/chan_bazalar.pdf.

Chen, C.S. (2002). Self-rgulated learning strategies and achievement in an introduction to information systems course. Information technology, learning, and performance journal, 2O(1), 11-25. Recuperado de http://search.proquest. com/openview/odof303f94687cofbcfc7a54ea8f4449/1?pq-origsite=gscholar.

Chun Chu, A. H., Choi, J. N. (2005). Rethinking procrastination: Positive effects of" active" procrastination behavior on attitudes and performance. The Journal of social psychology, 145(3), 245-264. doi: 10.3200/SOCP.145·3.245-264. 


\section{,}

Cirino Gerena, G. (2003). Los intereses como motivación intrínseca en la sala de clases. Perspectivas psicológicas, 3(4), 78-82. Recuperado de http://pepsic. bvsalud.org/pdf/pp/v3-4/v3-4ao8.pdf.

Colegio Colombiano de Psicología [COLPSIC]. (Marzo, 2012) Acuerdo numero 10 Por medio del cual se modifica el Manual Deontológico y Bioético de Psicología. Recuperado de http://www.colpsic.org.co/portal/tribunales_archivos/ ACUERDO_No_10_MANUAL_DEONTOLOGICO_Y_BIOETICO_DEL_PSICOLOGO_Marzo_15_2012.pdf o

Congreso de Colombia. (Octubre, 2012). Ley estatutaria 1581 de 2012. Por la cual se dictan disposiciones generales para la protección de datos personales. Recuperado de http://www.alcaldiabogota.gov.co/sisjur/normas/Norma1. jsp?i=49981.

Consejo Nacional de Acreditación (CNA) (2013). Lineamientos para la acreditación de programas de pregrado. Recuperado de http://www.cna.gov.co/1741/articles-186359_pregrado_2013.pdf.

Corno, L. (1994). Student volition and education: Outcomes, influences, and practices. Dale Schunk \& Barry Zimmerman (Eds.), (1994). Self-regulation of learning and performance: Issues and educational applications (pp. 229-251). Hillsdale, NJ, England: Lawrence Erlbaum Associates, Inc.

Cortina, M. (2003). Defensive processes, emotions and internal working models: a perspective from attachment theory and contemporary models of the mind. In: Cortina, M. \& Marrone, M. (2003). Attachment Theory and the Psychoanalytic Process. London: Whurr, pp. 307-335.

Dietz, F., Hofer, M., \& Fries, S. (2007). Individual values, learning routines and academic procrastination. The British journal of educational psychology, 77(4), 893-906. doi: 10.1348/000709906X169076.

Digdon, N.L., Howell, A.J. (2008). College students who have an eveningness preference report lower selfcontrol and greater procrastination. Chronobiology international, 25(6), 1029-1046. doi: 10.1080/07420520802553671. 
Domínguez Lara, S. A., Villegas García, G., Centeno Leyva, S. B. (2014). Procrastinación académica: validación de una escala en una muestra de estudiantes de una universidad privada. Liberabit, 2O(2), 293-304. Recuperado de http:// www.scielo.org.pe/pdf/liber/v2on2/a10v2on2.pdf.

Elliot, A. J., Church, M. A. (1997). A hierarchical model of approach and avoidance achievement motivation. Journal of personality and social psychology, 72(1), 218-232. doi://dx.doi.org/10.1037/0022-3514.72.1.218

Ferrari, J. R. (2001). Procrastination as self-regulation failure of performance: Effects of cognitive load, self-awareness, and time limits on 'working best under pressure'. European Journal of Personality, 15(5), 391-406.

Furlan, L. A., Heredia, D. E., Piemontesi, S. E., y Tuckman, B. W. (2013). Análisis factorial confirmatorio de la adaptación argentina de la escala de procrastinación de Tuckman (ATPS). Perspectivas en Psicología, 9(3), 142-149. Recuperado de http://www.seadpsi.com.ar/revistas/index.php/pep/article/view/111.

Glick, D. M., \& Orsillo, S. M. (2015). An investigation of the efficacy of acceptance-based behavioral therapy for academic procrastination. Journal of Experimental Psychology: General, 144(2), 400-409. doi: http://dx.doi.org/10.1037/ xgeo000050

Glick, D. M., Millstein, D. J., \& Orsillo, S. M. (2014). A preliminary investigation of the role of psychological inflexibility in academic procrastination. Journal of Contextual Behavioral Science, 3(2), 81-88. doi:10.1016/j.jcbs.2014.04.002

Gómez, A., y Rivas, F. (1997). Caracterización psicológica y operacionalización de la indecisión vocacional compleja. Iberpsicología, 2(2), 1-10. Recuperado de https://dialnet.unirioja.es/servlet/articulo? codigo=301210.

Good, T., Brophy, J.E. (1983). Motivación. En T. Good y J.E. Brophy: Psicología educacional. México: Interamericana.

Jackson, T., Weiss, K. E., Lundquist, J. J., \& Hooper, D. (2003). The impact of hope, procrastination, and social activity on academic performance of Midwestern 
college students. Education, 124(2), 310. Recuperado de https://www.questia. com/library/journal/1G1-112480005/the-impact-of-hope-procrastination-and-social-activity.

Kim, E., \& Seo, E. H. (2013). The relationship of flow and self-regulated learning to active procrastination. Social Behavior and Personality: an international journal, 41(7), 1099-1113. doi: http://dx.doi.org/10.2224/sbp.2013.41.7.1099.

Lepper, M. R. (1988). Motivational considerations in the study of instruction. Cognition and instruction, 5(4), 289-309. doi: 10.1207/s1532690xcio504_3.

Maytorena, M., González, D., y Velarde, D. (2006). Estrategias de aprendizaje y certeza vocacional en estudiantes de tres licenciaturas. En Congreso Mexicano de Psicología Social, XI (pp. 528-534).

Ministerio de Comercio, Industria y Turismo (Junio, 2013). Decreto Número 1317 de 2013. Por el cual se reglamenta parcialmente la Ley 1581 de 2012. Recuperado de www.mintic.gov.co/portal/604/articles-4274_documento.pdf.

Ministerio de Educación Nacional (MEN) (2010). Decreto No. 1295 de 2010 (20 de abril) por el cual se reglamenta el registro calificado de que trata la Ley 1188 de 2008 y la oferta y desarrollo de programas académicos de educación superior. Recuperado de http://www.mineducacion.gov.co/1621/articles-229430_ archivo_pdf_decreto1295.pdf.

Ministerio de Educación Nacional (MEN). (2014) Determinantes de la Deserción. Recuperado de http://www.mineducacion.gov.co/sistemasdeinformacion/1735/ articles-254702_Informe_determinantes_desercion.pdf.

Ministerio de Salud de la República de Colombia [MINSALUD] (1993) Resolución No. 8430 del 4 de Octubre de 1993, por la cual se establecen las normas científicas, técnicas y administrativas para la investigación en salud. Recuperado de http://issuu.com/scpbogota/docs/resolucion_8430.

Núñez, J. C., Cabanach, R. G., Rodríguez, S., González, J. A., y Rosário, P. (2009). Perfiles motivacionales en estudiantes de Secundaria: análisis diferencial en 
estrategias cognitivas, estrategias de autorregulación y rendimiento académico. Revista Mexicana de Psicología, 26(1), 113-124. Recuperado de http:// www.redalyc.org/pdf/2430/243016317011.pdf.

O’Brien, W. K. (2002). Applying the transtheoretical model to academic procrastination (Doctoral dissertation, ProQuest Information \& Learning).

Onwuegbuzie, A. J. (2004). Academic procrastination and statistics anxiety. Assessment \& Evaluation in Higher Education, 29(1), 3-19. doi: 10.1080/0260293042000160384.

Pérez R, A (2007). Los procesos de exclusión en el ámbito escolar: el fracaso escolar y sus actores. Revista Iberoamericana de Educación, 43(6), 1-9. Recuperado de https://dialnet.unirioja.es/servlet/articulo?codigo=2358611.

Pérez, A., Ramón, J., y Sánchez, J. (2000). Análisis exploratorio de las variables que condicionan el rendimiento académico. Sevilla: Universidad Pablo de Olavide.

Pérez, J. C. N., y Cabanach, R. G. (1998). Variables cognitivo-motivacionales, enfoques de aprendizaje y rendimiento académico. Psicothema, 1O(2), 393-412. Recuperado de http://www.psicothema.com/pdf/173.pdf.

Pintrich, P. R. (2004). A conceptual framework for assessing motivation and self-regulated learning in college students. Educational Psychology Review, 16(4), 385-407. doi: 1040-726X/04/1200/0385/o.

Pintrich, P.R., \& De Groot, E.V. (1990). Motivational and self-regulated learning components of classroom performance. Journal of Educational Psychology, 82(1), 33-40. doi: //dx.doi.org/10.1037/0022-0663.82.1.33.

Pychyl, T. A., Lee, J. M., Thibodeau, R., \& Blunt, A. (2000). Five days of emotion: An experience sampling study of undergraduate student procrastination. Journal of social Behavior and personality, 15(5), 239-254. Recuperado de http://search.proquest.com/openview/7e3502c28fbo6c9517ccbe6eo8f$\mathrm{d} 637 \mathrm{e} / \mathrm{1}$ ?pq-origsite $=$ gscholar\&cbl=1819046. 
Quant, D. M., Sánchez, A. (2012). Procrastinación, procrastinación académica: concepto e implicaciones. Revista Vanguardia Psicológica Clínica Teórica y Práctica, 3(1), 45-59. Recuperado de http://apps.umb.edu.co/revp/index. php/vanguardiapsicologica/article/viewArticle/63.

Robbins, S. B., Morrill, W. H., \& Boggs, K. (1987). The construction and validation of the Career Factors Inventory. Manuscrito no publicado, Virginia Commonwealth University, Richmond.

Sánchez, F., Quirós, M., Reverón, C., y Rodríguez, A. (2002). Equidad Social en el Acceso y Permanencia en la Universidad Pública. Determinantes y Factores Asociados. Centro de Estudios para el Desarrollo Económico (CEDE), Bogotá: Universidad de los Andes. Recuperado de http://www.zef.de/module/register/media/12ff_ACCESO\%20Y\%20PERMANENCIA\%2OUNIVERSIDADES\%2oESPA\%C3\%91OL.pdf.

Schiefele, U. (1991). Interest, learning, and motivation. Educational psychologist, 26(3-4), 299-323. doi: 10.1080/00461520.1991.9653136.

Schwinger, M., Steinmayr, R., \& Spinath, B. (2009). How do motivational regulation strategies affect achievement: Mediated by effort management and moderated by intelligence? Learning and Individual Differences, 19(4), 621-627. doi:10.1016/j.lindif.2009.08.006.

Steel, P. (2007). The nature of procrastination: a metaanalytic and theoretical review of quintessential selfregulatory failure. Psychological bulletin, 133(1), 65-94. doi: //dx.doi.org/10.1037/0033-2909.133.1.65

Steel, T., Brothen, T, \& Wambach, C. (2001). Procrastination and personality, performance, and mood. Personality and Individual Differences, 30, 95-106. doi: 10.1016/So191-8869(oo)ooo13-1.

Tice, D. M., \& Baumeister, R. F. (1997). Longitudinal study of procrastination, performance, stress, and health: The costs and benefits of dawdling. Psychological science, 8(6), 454-458. Recuperado de http://www.jstor.org/stable/40063233. 
Tuckman, B.W. (1990). Group versus goal-setting effects on the selfregulated performance of students differing in self-efficacy. Journal of Experimental Education, 58(4), 291-298. doi: 10.1080/00220973.1990.10806543.

Universidad Pedagógica y Tecnológica de Colombia (Uptc) (2011). Diagnóstico institucional a la deserción estudiantil. Recuperado de http://virtual.uptc.edu.co/ acreditacion/MODELO/ANEXOS/FACTOR_ESTUDIANTES/DIAGNOSTICO_INSTITUCIONAL_DESERCION.pdf.

Universidad Pedagógica y Tecnológica de Colombia [Uptc] (1998). Acuerdo No. 130 de 1998 (22 De Diciembre) por el cual se expide el Reglamento Estudiantil. Recuperado de https://registro.uptc.edu.co/estudiantes/documentos/reglamento_uptc.pdf

Valle, A., González, C. Nuñez, P. Martínez, R. Pineñor, A. (1999). Un modelo causal sobre los determinantes cognitivo motivacionales del rendimiento académico. Revista de psicología general aplicada, 52(4), 499-519.

Wolters, C. A., \& Pintrich, P. R. (1998). Contextual differences in student motivation and self-regulated learning in mathematics, English, and social studies classrooms. Instructional science, 26(1), 27-47. doi: 10.1023/A: 1003035929216. 